\title{
A Computational Model for Tumor Cell Membrane Tolerance and Rigidy Limits
}

\author{
George I. Lambrou, Apostolos Zaravinos, Maria Adamaki, Dimitrios Delakas, Spiros Vlahopoulos
}

\begin{abstract}
Cancer is one of the leading fatal diseases in the western world and many approaches have been proposed for both its treatment and prevention. Several of these approaches are dealing with the use of chemicals or with the use of signaling molecules that interfere with critical pathways in cancer progression. Yet, there is still a lot to be learned for the biology and mechanisms of oncogenesis, tumor progression, and tumor biology in general. What that has been neglected throughout the years is the understanding of biological systems from the physical perspective and the understanding of their mechanisms from the physical point of view. Since living systems could be considered as physical systems, there exists the possibility of examining biological phenomena under this perspective. In the present work we use computational and physical approaches to study and model the tolerance limits and mechanics of cell membranes. Our scope is to gain a better understanding of these phenomena and potentially use them for therapeutic purposes, since we are referring to tumor cells.
\end{abstract}

\section{INTRODUCTION}

$\mathrm{C}$ ancer is one of the leading fatal diseases in the western world. Its treatment is of major importance not only because of its high mortality rate but also due to the devastating effect it has on the quality of life of the affected individual. In addition, it has been shown that due to the complexity of the disease it is essential to create patientspecific therapies and not generally applicable ones. In addition, it might be possible that tumors have a common Achilles' heel and so a common treatment could become feasible. As a consequence, there is a growing interest for methods that would allow treatment, i.e. intervention against the tumor, without the invasiveness that the classical therapies confer. For this to be achieved, it is important to gain a more in-depth understanding of the physical properties of tumor cells. This would set the framework for further investigations, in particular for treating tumor cells with the help of mechanical means, as for example shock

Manuscript received July 5, 2001.

GIL is with the $1^{\text {st }}$ Department of Pediatrics, University of Athens, Choremeio Research Laboratory, Thivon \& Levadeias, 11527, Goudi, Athens, Greece (+302107467427, e-mail: glamprou@med.uoa.gr)

AZ is with the Department of Clinical Virology, University of Crete, School of Medicine, 71110, Heraklion, Crete (e-mail: azaravinos@gmail.com)

MA is with the $1^{\text {st }}$ Department of Pediatrics, University of Athens, Choremeio Research Laboratory, Thivon \& Levadeias, 11527, Goudi, Athens, Greece (e-mail: madamaki@med.uoa.gr)

DD is with the Department of Urology, Asklipieio General Hospital, 16673 Voula, Athens, Greece (email: Delakas@mail.gr)

$\mathrm{SV}$ is with the $1^{\text {st }}$ Department of Pediatrics, University of Athens, Choremeio Research Laboratory, Thivon \& Levadeias, 11527, Goudi, Athens, Greece (e-mail: sblachop@med.uoa.gr) waves, as these have been widely used in the treatment of bile stones and ultra-sonic lithotripsy. The lipid bilayer has been initially recognized as the basic architectural form of cell encapsulation $[1,2]$. Later on models of cell membranes have been reported, with the fluid-mosaic model constituting the most prevalent one [3]. Cell membrane elasticity appears to be a cell-specific property [4] and as a consequence the effects of shock waves could also be cell-specific. An older report by Brummer et al. (1989) demonstrated that shock waves from electrical sparks have an effect on leukemic cells [5]. This finding suggests that, first of all there is a great of lack of knowledge as far as the mechanics of cells are concerned, and second, if we assume that there is a possibility to model such phenomena, then this could have a positive effect on tumor therapies. The scope of the present work is to set a computational and theoretical framework for the study of tumor cell mechanics, with emphasis given on cell membrane tolerance and rigidy.

\section{THEORETICAL FRAMEWORK}

\section{A. Mathematical Formulations}

Cell membrane mechanics can be determined by two main mechanisms: lipid-bilayer interactions between the amphiphilic molecules constituting it, and the underlying structure of actin, spectrin and tropomyosin filaments. Therefore, we could assume that there are several forces holding the membrane $\left(F_{\text {total }}\right)$, i.e. the cell, together, as it is presented in Fig. 1. There are four types of forces that can be accounted for holding the cell membrane, i.e. the cell, intact: $\Sigma F_{C}$, Coulomb forces, $\Sigma F_{L}$, electromagnetic Laplace forces, $\Sigma F_{M}$, mechanical forces and $F_{H}$, hydrostatic pressure exerted on cell membranes due to the surrounding aqueous environment. In addition, there are several other forces that hold the cell membrane together, as for example there is evidence that electrostatic forces play a role between the lipid bilayer and the cytoskeleton [6]. Yet, for simplicity reasons we will consider the former four forces in our study. Therefore, the sum of all forces will be,

$\sum \vec{F}=\sum \vec{F}_{C}+\sum \vec{F}_{L}+\sum \vec{F}_{M}+\sum \vec{F}_{H}$

The mechanical forces $\left(F_{M}\right)$ can be derived only experimentally and they vary between different cell types. However, mechanical forces can be evaluated if we consider a simple model for the cell membrane structural mechanics, which is presented in Fig. 2. What we observe is a triangular structure consisting of three main proteins that hold cell membrane together, such as actin, spectrin and tropomyosin. In that formation 


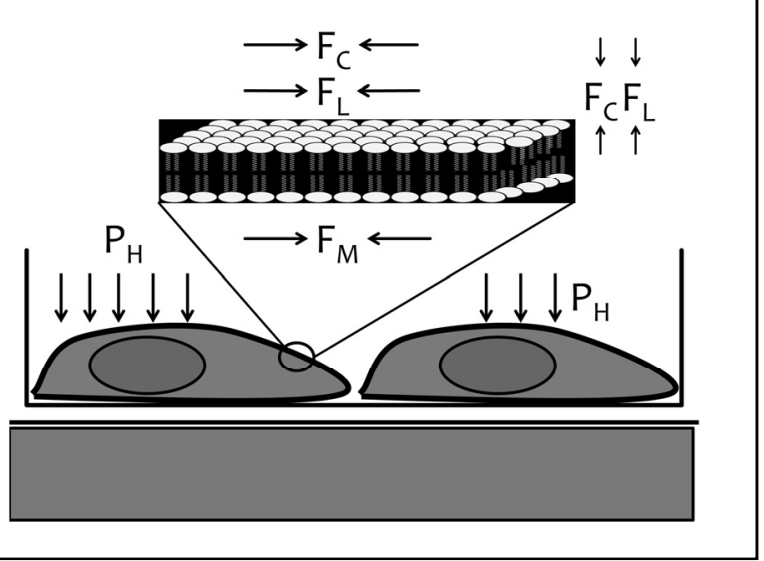

Fig. 1. A simple representation of cells grown in culture. The forces exerted on cells are presented, where $F_{C}$ is Coulomb forces, $F_{L}$ is Laplace forces mainly due to inter-molecular forces, $F_{M}$ are the mechanical forces exerted mainly due to cytoskeletal proteins and $\mathrm{P}_{\mathrm{H}}$ is the hydrostatic pressure exerted on cells due to the overlying fluids.

$$
\Sigma F_{m}=0
$$

Hydrostatic forces can be calculated using Pascal's Law

$$
P_{P}-P_{0}=\rho g\left(h-h_{0}\right)
$$

where $P_{P}, P_{0}$ are the pressures exerted at a point $p$ and a reference point respectively, is the fluid's density and $h$ is the height difference from a reference point. Finally, for the electromagnetic forces, Maxwell's equation for electromagnetism could be utilized, since these include the electric field from electrostatic charges as well as electromagnetic charges. Hence, the total electric field produced would be

$$
\vec{E}_{\text {total }}=\vec{E}_{C} \times \vec{E}_{L}
$$

According to Maxwell's equations this can be translated as

$\oint B d l=\varepsilon_{0} \mu_{0} \frac{d}{d t} \int E d A$

and $\oint E d l=-\frac{d}{d t} \int B d A$

We will consider Laplace forces as negligibly small and hence,

$$
\vec{E}_{L}=0
$$

Therefore,

$$
\vec{E}_{\text {total }}=\vec{E}_{C}
$$

The electric field created by a point charge is

$$
E(x)=\frac{q}{4 \pi \varepsilon_{0} x^{2}}
$$

and the work required to move a unit charge from the reference point is

$$
\Psi(x)=\int_{r e f}^{x} E d x \quad(10),
$$

which equals to the energy required to disrupt the membrane. Given that the cell membrane has an electric

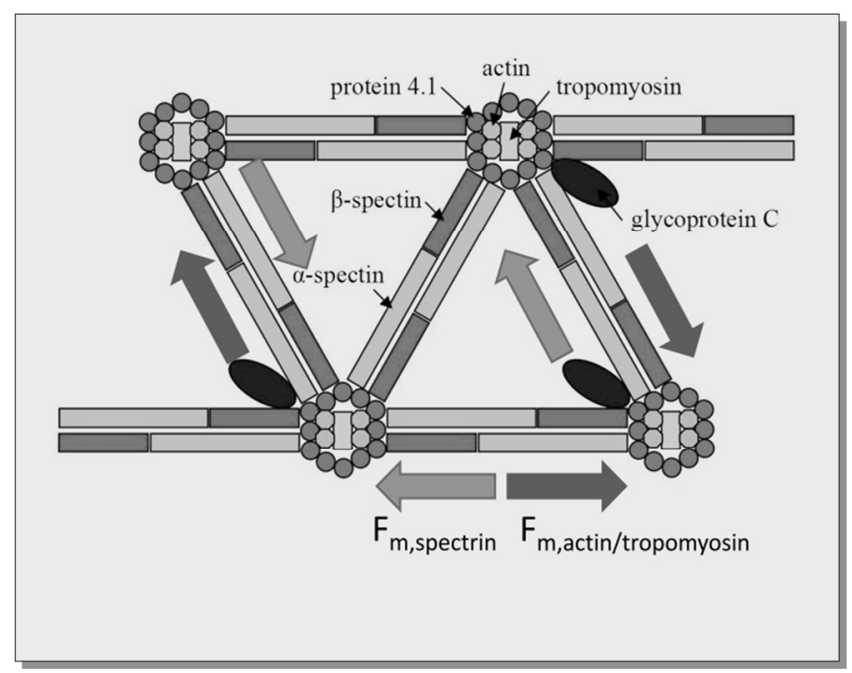

Fig. 2. The structural arrangement of the cytoskeleton. The forces are represented with arrows, indicating the opposite forces exerted by spectrin and actin/tropomyosin respectively.

field, then it also has a potential $\Psi$, that it is equal to the energy required to move a particle away from it. Using the Poisson's and Gouy-Chapman formulations, for surface charges we get

$$
\frac{q_{s}}{\varepsilon \varepsilon_{0} \Psi_{0}}=\sqrt{\frac{2 C_{0} \alpha F^{2}}{R T \varepsilon \varepsilon_{0}}}
$$

which connects the charge of a surface $\left(q_{v}(x)\right)$ to the surface's potential $(\Psi(x))$, where $q_{s}$ is the charge of the surface, $\varepsilon$ and $\varepsilon_{0}$ are the dielectric constants for the membrane and vacuum respectively, $C_{0}$ is the concentration of ion species in moles/lt, $F$ is the Faraday constant, $\alpha$ is the constant $10^{3} \mathrm{l} / \mathrm{m}^{3}, R$ is gas constant and $T$ is the temperature.

The final and probably the most complex forces are the mechanical forces that hold the membrane cells together. Actin and tubulin filaments form a network of "chains" that make up the structural architecture of the cell. In order to understand their role, simulations would be required, since each protein can be considered as a spring and the sum of proteins as a network of interconnected springs. To simplify our analysis we consider the net force of the cytoskeleton.

For the cell membrane to be disrupted, forces should be exerted on it; in the present case these would be derived from shock waves $(s w)$ or any other source of energy transmission, such that

$\sum F_{S W}>\sum F_{\text {total }}$

The work required to disrupt the cell membrane would be equal to the work required to move parts of charged membrane apart from each other to infinite distance. Hence,

$d W_{\text {total }}=-\int_{0}^{+\infty} \sum F_{\text {total }} d x$

When at equilibrium, this simplified system is

$\Sigma F_{\text {total }}=0$

This means that the pressure exerted on cells equals the force exerted by the cells to the surrounding fluid. 


\section{B. Biological Considerations}

From a biological point of view, cell rigidy is a complex subject. A thought that came to us when considering our model for cell membranes is the following: in in vitro cell cultures adherent cells reside on the plate's surface with a fluid culture medium surrounding the cells. If a cell has, let's say, a size of $10 \mathrm{um}$ and the fluid above is of a depth of, let's say, $50 \mathrm{~mm}$, then the hydrostatic pressure would be approximately equivalent to a human being submerged to a pressure at a depth of $700 \mathrm{~m}$ below the sea surface. By taking this fact into account, the forces exerted on the cells in the medium must be immense compared to their size. This means that the forces holding the cells together are also very strong, since they do not rupture. As a matter of fact, not only do they not rupture, but they are also able to proliferate under these conditions and even to perform several molecular functions.

The question arising here is whether it is possible for cells to be different in their mechanical properties. When it comes to cancer cells, it has been reported by other groups that they are more abundant in actin and tubulin proteins than other cells, since they require these proteins in order to proliferate and to migrate from their original site to distant sites when metastasizing. In other words, these observations obtained by experimentation, now acquire new meaning and are being considered from the physical point of view.

Nature itself exploits this property of cells in order to induce apoptosis. A known mechanism of apoptosis includes the activation of caspases, a group of proteolytic enzymes that cleave the filaments holding together the cell, which subsequently causes cell death.

\section{SimULATIONS}

As we mentioned above, in order to cause a disruption of the cell membrane, we assume the following to be true:

$$
d W_{\text {total }}=-\int_{0}^{+\infty} \sum F_{\text {total }} d x
$$

and

$$
\sum F_{S W}>\sum F_{\text {total }}
$$

Based on the previous formulations, we could define the total forces holding the membrane. Therefore,

$$
\sum \vec{F}_{S W}>\vec{F}_{C}+\vec{F}_{M}
$$

Also, the total work required would be equal to the sum of the works required for each force such as:

$$
d W_{\text {total }}=\int_{0}^{+\infty} \sum F_{C} d x+\int_{0}^{+\infty} \sum F_{M} d x
$$

and consequently,

$$
W_{\text {total }}=W_{C}+W_{M}=\Psi(x)+W_{M}=\frac{q_{S}}{\varepsilon \varepsilon_{0} \sqrt{\frac{2 \Psi_{0} a F^{2}}{R T \varepsilon \varepsilon_{0}}}}+\int_{0}^{+\infty} \sum F_{M} d x
$$

In order to specify our hypothesis further, the mechanical force that should be exerted on the cell is regarded as the unknown factor. Its magnitude would depend upon the electrostatical properties of the cell and mainly on the tolerance limits of the cytoskeletal filaments. From equation (19) we understand that there are few factors that can change. The first is of course the mechanical force that should be exerted. The second is the surficial charge $q_{s}$ and the third is the concentration of the ions $C_{0}$, considering that the effects are taking place at constant temperature. Another factor that can change is the dielectric constant $\varepsilon$, which depends on the phospholipid layer. This factor can also be regarded as a constant, since the main constituents of the membrane are phospholipids. We can name the $\varepsilon \varepsilon_{0}$ factor as the total membrane dielectric $\left(\varepsilon_{m}\right)$, such as

$\varepsilon_{m}=\varepsilon \varepsilon_{0} \quad(20)$.

Also, we could name the remaining constant factors as:

$$
k=\frac{2 \alpha F^{2}}{R T}
$$

Hence, equation (19) becomes:

$$
W_{\text {total }}=\frac{q_{S}}{\varepsilon_{m} \sqrt{\frac{\kappa}{\varepsilon_{m}} C_{0}}}+\int_{0}^{+\infty} \sum F_{M} d x
$$

The work performed by a force $\mathbf{F}$ is defined as

$W=\boldsymbol{F} \cdot x=F x \cos \theta$

Equation (22) becomes,

$$
W_{\text {total }}=\frac{q_{S}}{\varepsilon_{m} \sqrt{\frac{k}{\varepsilon_{m}}} C_{0}} F x \cos \theta
$$

We can simulate equation (24) by keeping as constants the variables one by one. We have used the Matlab ${ }^{\circledR}$ (The Mathworks Inc.) simulating environment for further simulations. We have used experimental data from other reports in order to set the range for values that cannot be calculated, as for example the force $\mathbf{F}$ [7]. In Fig. 3 we present the total work with respect to the total mechanical force and the surficial charge, the mechanical force and the ion concentration, and the surficial charge and ion concentration respectively. Especially in the first case where the total work is presented as a function of the mechanical forces and the surficial charge, an interesting notion was made of the three variables forming a "saddle", which could be explained as a precursor of non-linearity.

\section{DISCUSSION}

In the present work we have attempted a preliminary approach to the physical properties of cell membranes with respect to their tolerance and rigidy. These findings could prove useful as alternative therapies in neoplastic diseases, and tumors could be studied further by exploiting the properties of cell membranes. Not much is known on the mechanics of cell structure and this could become a promising field for future therapeutic design. Previous 
reports have demonstrated that exerting forces on cells, which is essentially a mechanical stimulus, could stimulate molecular and phenotypical alterations such as proliferation. This has been reported for fibroblasts [8] and for leukemia cells, where it was noted that mechanical forces exerted inhibitory effects [5]. The idea, in general, of exploiting cell membrane tolerance for therapeutic reasons is not new. In previous reports Sonodynamic therapy (SDT) has been reported as a possible treatment for tumors by Umemura et al. (1989) when they studied synergistic effects of ultrasound (US) and hematoporphyrin (HPD) [9]. The same technique has been utilized later in order to activate enzymes on the cell membrane showing that plasma membrane fluidity can affect biological processes [10]. In another interesting work it has been reported, supporting the present ideas, that plasma membrane fluidity can affect the way tumor cells react to anti-tumor agents [11]. In another work it is supported that tumor cells do possess membranes with different physical properties as compared to normal cells [12]. It has been reported that certain chemotherapeutic agents can induce plasma membrane alterations, thus stimulating apoptosis $[13,14]$. The opposite is also true as changes in cell membrane fluidity can cause effects such as resistance to therapeutic agents. In conclusion, these are cellular traits that can be exploited in order to develop new alternative therapies to cancer. There is still a lot to be learned on biological systems and their physical properties, and further interdisciplinary investigations are required in order to gain more knowledge in the particular field.

\section{REFERENCES}

[1] H. Davson and J. F. Danielli, "Studies on the permeability of erythrocytes: The alleged reversal of ionic permeability at alkaline reaction," Biochem J, vol. 30, Feb 1936, pp. 316-20.

[2] E. Gorter and F. Grendel, "On Bimolecular Layers of Lipoids on the Chromocytes of the Blood," J Exp Med, vol. 41, Mar 31 1925, pp. 439-43.

[3] S. J. Singer and G. L. Nicolson, "The fluid mosaic model of the structure of cell membranes," Science, vol. 175, Feb 18 1972, pp. $720-31$.

[4] I. Titushkin and M. Cho, "Regulation of cell cytoskeleton and membrane mechanics by electric field: role of linker proteins," Biophys J, vol. 96, Jan 2009, pp. 717-28.

[5] F. Brummer, J. Brenner, T. Brauner, and D. F. Hulser, "Effect of shock waves on suspended and immobilized L1210 cells," Ultrasound Med Biol, vol. 15, 1989, pp. 229-39.

[6] E. Kahana, S. Streichman, and B. L. Silver, "The role of electrostatic forces in the interaction between the membrane and cytoskeleton of human erythrocytes," Biochim Biophys Acta, vol. 1066, Jul 1 1991, pp. $1-5$.

[7] D. R. Overby, B. D. Matthews, E. Alsberg, and D. E. Ingber, "Novel dynamic rheological behavior of individual focal adhesions measured within single cells using electromagnetic pulling cytometry," Acta Biomater, vol. 1, May 2005, pp. 295-303.

[8] E. A. Schwartz, R. Bizios, M. S. Medow, and M. E. Gerritsen, "Exposure of human vascular endothelial cells to sustained hydrostatic pressure stimulates proliferation. Involvement of the alphaV integrins," Circ Res, vol. 84, Feb 19 1999, pp. 315-22.

[9] N. Yumita, R. Nishigaki, K. Umemura, and S. Umemura, "Hematoporphyrin as a sensitizer of cell-damaging effect of ultrasound," Jpn J Cancer Res, vol. 80, Mar 1989, pp. 219-22.

[10] W. Tang, Q. Liu, X. Wang, N. Mi, P. Wang, and J. Zhang, "Membrane fluidity altering and enzyme inactivating in sarcoma 180
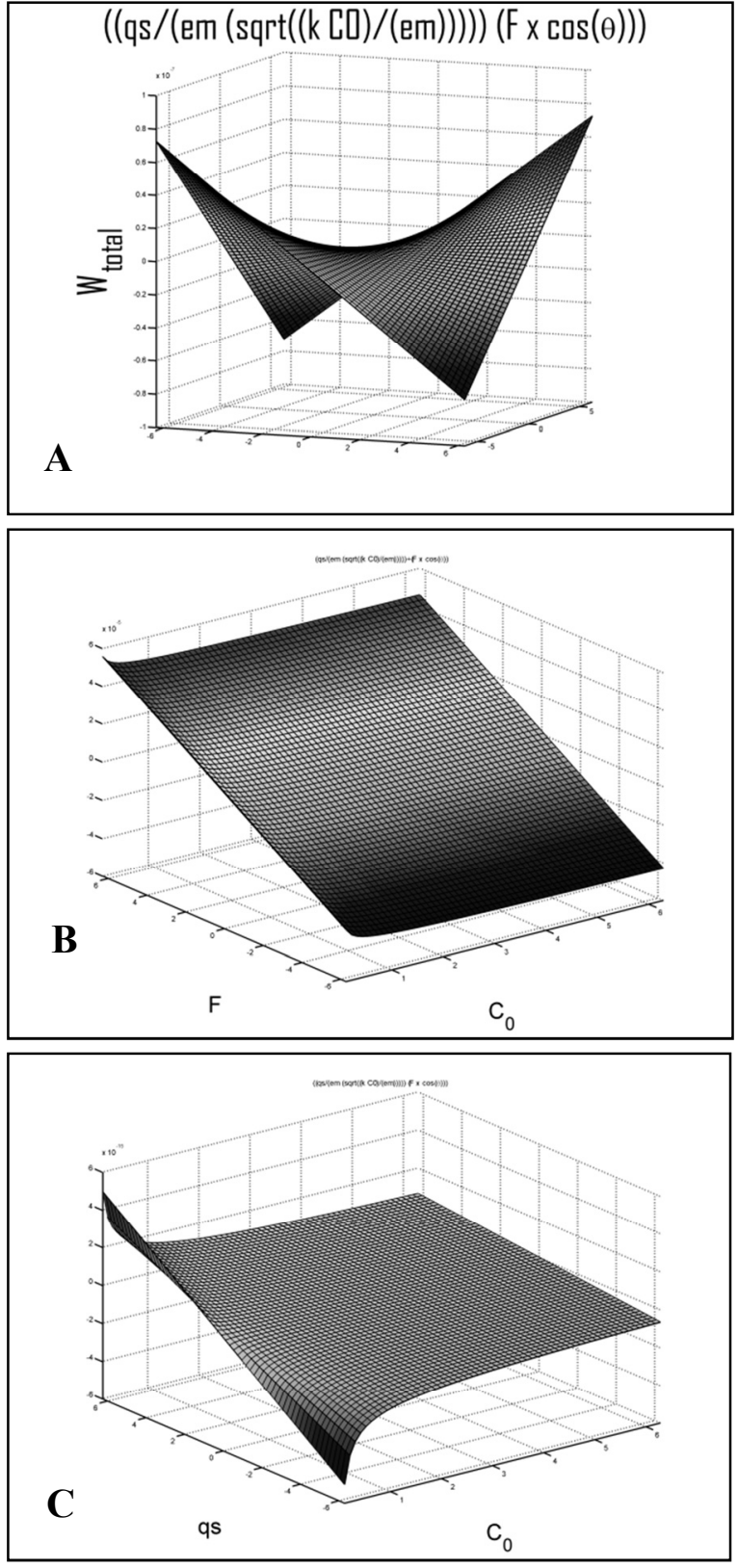

Fig. 3. Simulations of the total work needed for cell membrane disruption with respect to the total exerted mechanical force and surficial charge (A), the total exerted mechanical force and membrane ion concentration (B) and surficial charge and membrane ion concentration $(\mathbf{C})$.

cells post the exposure to sonoactivated hematoporphyrin in vitro," Ultrasonics, vol. 48, Mar 2008, pp. 66-73.

[11] S. Baritaki, S. Apostolakis, P. Kanellou, M. T. Dimanche-Boitrel, D. A. Spandidos, and B. Bonavida, "Reversal of tumor resistance to apoptotic stimuli by alteration of membrane fluidity: therapeutic implications," Adv Cancer Res, vol. 98, 2007, pp. 149-90.

[12] T. Galeotti, S. Borrello, G. Minotti, and L. Masotti, "Membrane alterations in cancer cells: the role of oxy radicals," Ann N Y Acad Sci, vol. 488, 1986, pp. 468-80.

[13] N. Aouali, L. Eddabra, J. Macadre, and H. Morjani, "Immunosuppressors and reversion of multidrug-resistance," Crit Rev Oncol Hematol, vol. 56, Oct 2005, pp. 61-70.

[14] D. K. Perry, "The role of de novo ceramide synthesis in chemotherapy-induced apoptosis," Ann N Y Acad Sci, vol. 905, Apr 2000, pp. 91-6. 